\title{
In Situ, Operando Measurements of Rechargeable Batteries
}

\author{
Howard Wang ${ }^{1, *}$, Feng Wang ${ }^{2}$ \\ ${ }^{1}$ Department of Materials Science and Engineering, University of Maryland, College Park, MD \\ 20742, USA \\ ${ }^{2}$ Sustainable Energy Technologies Department, Brookhaven National Laboratory, Upton, NY, \\ 11973, USA
}

\begin{abstract}
This article reviews recent in operando measurements (IOMs) for addressing challenges in advancing rechargeable battery $(\mathrm{RB})$ technologies. As the demands on energy and power density of RBs for broader applications continue to grow, current RB technologies are pushed to their theoretical and engineering limits while new approaches are being extensively investigated. IOMs have become more powerful and effective research tools in recent years; they will play an essential role in developing next generation RBs. This review is organized around outstanding issues in battery science and engineering. We emphasize the critical need for quantifying the distribution and transport of active ions in functioning batteries over wide temporal and spatial scales in real time.
\end{abstract}

*Corresponding author:

Howard Wang, wangh@umd.edu, 4418 Stadium Dr., College Park, MD 20742, USA. Phone: 013014050005, fax: 01-3013142029 
Graphical Abstract:

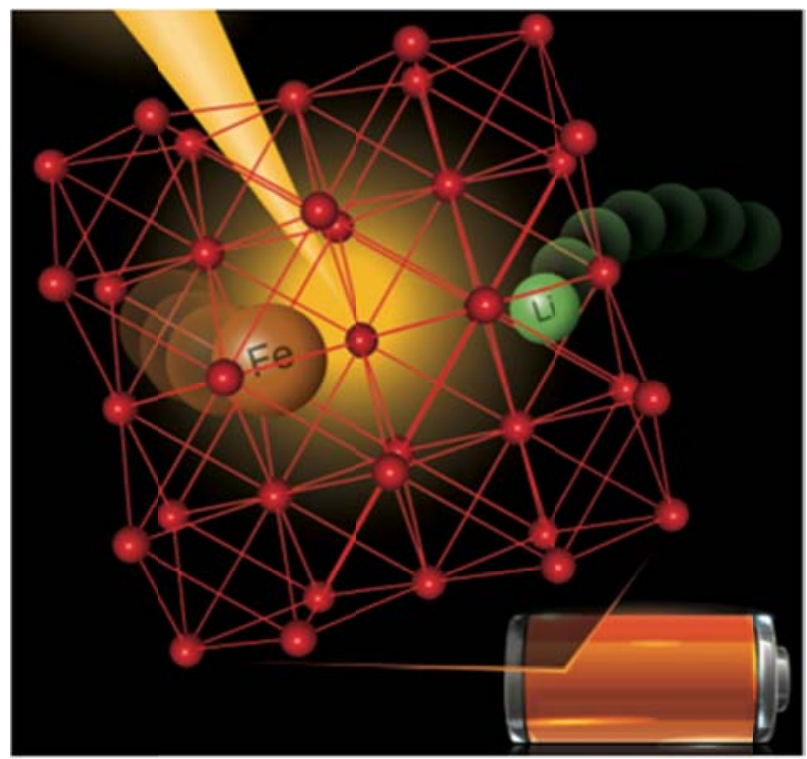




\section{Introduction: Challenges and Needs}

Rechargeable batteries (RBs) are indispensable in the modern society; they will play a bigger role in the future. Today, lithium ion batteries (LIBs) are the preferred energy storage technology for portable electronics; their uses in plug-in and hybrid electric vehicles and distributed renewable energy systems are rapidly increasing. [1, 2] However, the energy storage density of most commercial cells is still low, an increase of energy density per unit cost by two times or more is needed for widespread and large-scale applications. Despite the extensive effort invested in searching for new materials to elevate the energy storage capacity, in the immediate future, continuous effort is needed to improve the use of existing battery materials and architectures, particularly through better understanding and control of the local ionic and electronic transport.

Often depicted as three stacked layers of cathode, anode and electrolyte, RBs are actually complex, typically use composite electrodes consisting of electric and ionic transport pathways connecting active particulates of nano- to micron-size, with $\AA$-dimension lattices orchestrating ion/electron flows to facilitate battery operations [as illustrated in Fig. 1(a)]. [3,4] Most RBs operate with a "rocking-chair" mechanism, in which active ions move back and forth between the cathode and anode in charge/discharge cycles. As individual charge transfer occurs over a unit lattice cell space in picoseconds, a battery package may need to meet a product specification requirement of lasting for a decade and performing a thousand charge/discharge cycles. The RB reliability over wide range of length and time scales needs to be maintained amidst dynamic interplays of many physical and chemical processes, which are intrinsically non-equilibrium and transient.[3] The key to realizing reproducible battery operations is the robustness of three interconnected networks, electric, ionic and mechanical, superimposed on one another. The 
mechanical network imposes elastic restoring forces that hold electrode materials in place and maintain the integrity of both electric and ionic transport networks that facilitate the concurrent flows of ions and electrons to/from each active particle. [5**, $\left.6^{*}\right]$

Better fundamental understanding of the complexity and operating mechanisms in RBs is essential to addressing outstanding challenges, such as high energy and power density, safe to use, long cycle life, high efficiency, and special application requirements, to list just a few. A common theme underlying the grand challenges is the distribution and transport of active ions in electrodes and across interfaces. Conventional electrochemical measurements reveal basic information about how a battery behaves, in operando measurements (IOMs) and ionic quantifications often could answer why it behaves that way. Research using IOMs has enjoyed rapid advances in techniques in the past decade, and many of those are described in recent review papers and book chapters.[7,8,9,10*] In research literatures, in situ refers to a specimen "on site" of its chemical and physical environment and in operando refers to operational and functional states; they are often used interchangeably in describing measurements of RBs during charge/discharge. We follow the convention of mixed usages while emphasizing operational and functional aspects in RB research. This short review focuses mainly on the latest development of in situ, operando measurement techniques and their applications to addressing major challenges in advancing battery technologies.

\section{Overview of In Operando Measurements}

To achieve the goal, different IOM techniques are used to obtain essential knowledge of structure, morphology, composition and bond states of metals (as redox centers) in electrodes over wide length and time scales during battery operation, as exemplified in Fig. 1(b). 
Conventional techniques have been continuously improved to afford measurements on the bulk of electrodes with wider dynamic ranges and higher resolutions. Recent advances have focused on IOMs at very small scales, on individual particles in their local environment. As both the bulk and single particle measurements are essential, and will be pursued further, tools for revealing the collective behavior of many body particulates with intertwined and interdependent physiochemical processes are still lacking.

As the complexity due to nonlinear feedback and stochastic processes cannot be quantified exactly, it is often assessed by characteristic quantities. We consider the concentration of active ions at any position and time, $c_{\mathrm{i}}(\overrightarrow{\boldsymbol{r}}, t)$, the essential parameter for describing a battery system. Several recent developments of IOMs align with this view, through the innovative use of transmission electron microscopy (TEM), [10,11,12,13,14,15] scanning probe microscopy (SPM),[16,17] nuclear magnetic resonance (NMR) spectroscopy,[18] and synchrotron x-ray diffraction (XRD),[6,19,20,21,22] as well as neutron scattering and activation. $\left[8^{*}, 23^{*}, 24,25\right]$ Applications of neutron techniques for in situ diagnosis are increasing; they can be very powerful because of neutron's high penetration power and relatively high sensitivity to $\mathrm{Li}$ isotopes.

Advanced IOMs offer new opportunities for in-depth studies of ion transport, electrochemical reactions, and phase transformations at wide length scales, from electrodes to single particles and interfaces, and time scales, from hours to milli- and picoseconds (Fig. 2), enabling direct comparison with theories and simulations.[3] Furthermore, two or more techniques are often combined to provide multimodal analysis, allowing for deconvolution of multiple interacting processes that occur at electrodes, electrolytes and interfaces. [26,27,28] 
Major battery issues are critically related to the location and transport of active ions. Specifically, (1) the energy density is determined by valence states and the site occupancy, which are tied to the working potential and the theoretical charge storage capacity, respectively; (2) the power density is limited by the pathway and efficiency of ionic transport in electrode and electrolyte layers; (3) the safety and life time of RBs depend largely on the control of undesirable ion depositions and irreversible parasitic reactions. In principle, all essential electrochemical processes in RBs are attributed to the dynamic interplay between active ions and electrons in their local environment. In the following, advances and needs of IOMs for major challenges in the current and next generation RBs are discussed.

\section{High Energy Density}

The primary research pursuit on RBs is high energy density, in both gravimetric and volumetric measures, through increasing the operation voltage, or the charge storage capacity, or both. [Error! Bookmark not defined.,4,29,30*,31*] The latter has led to electrodes operated with conversion reactions, which will be discussed a later section of this review. The former has resulted in high voltage layered intercalation cathodes that will likely dominate the market in the near future. Here, measurement issues emerge in two distinct research directions, (1) toward higher intrinsic or theoretical capacities, and (2) toward better utilization of the limits, in accord to research in materials chemistry and engineering, respectively. In the former, spectroscopic IOMs are used to determine valence states and diffraction for site filling, resulting in atomic level understanding of the structure, location and occupancy of ionic sites for assessing the true energy storage capacity and phase stability.[11, 26, 32] Selected examples are shown in Fig. 3. 
In addition to quantifying the entire electrode, novel IOMs have been developed to suite for single particle measurements.[22,12,13,14] Tracking ionic/electronic transport in single crystalline particles by synchrotron X-ray and TEM has opened the door for assessing battery materials at the most fundamental level, initially at the cost of missing the important information of the linkage and interactions among particles. In IOMs at the single-particle level, in addition to seeing lattice structures and ionic occupancy and flow in the target particle, information gathering can also be extended to understanding the structure and role of the surface and interfaces, which play a critical role in battery performance as they indicate the particle stability and mediate the ion transport among active particles and through the matrix. Those are intimately related to the engineering aspect of the problem in achieving the theoretical capacity, which can only be asymptotically approached in practice.

The engineering challenge is the design, fabrication and perseverance of the interconnected electrical, ionic and mechanical networks to allow for simultaneous transport of ions and electrons through low-resistance and continuous networks to reach each active particle in a battery and maximize the usable energy capacity. In traditional batteries using randomly distributed conductive phases among active materials, transport heterogeneity always exists; perfect wiring is not achievable.[5**,7] It remains unclear whether the heterogeneity is the intrinsic nature of electrochemistry and thermodynamics in batteries, or it can be overcome by engineering means.

Furthermore, the connectivity and conductance of transport networks evolve continuously as the composition and thickness of solid-electrolyte interphase (SEI) vary with charge/discharge cycles, or even when the battery sits idle.[33] The needs to understand and control the transport heterogeneity and its evolution pose the ultimate challenge to IOMs for batteries. Mapping ionic 
current density in 3D over all relevant length and time scales, superimposed on the corresponding structural and compositional maps, remains the pinnacle goal of IOMs. As the task appears insurmountable in the foreseeable future, the current focus is on developing IOMs capable of measuring multiple interacting particles under the same conditions as in real electrodes. Studies toward that direction have been limited, but more are expected.[26, 8, 9, 34]

\section{High Power Density}

Increasing demands in power electronics push the envelope of the battery power density at both the device and packaging levels.[31] The latter is the subject of electrical and mechanical engineering, whereas battery research focuses on materials and cells and the transport of active ions therein. The key capability of IOMs for addressing the power density issues is real-time tracking of ions moving within electrode particles, across surfaces and SEIs, and throughout electrode layers, i.e., $c_{\mathrm{i}}(\overrightarrow{\boldsymbol{r}}, t)$, at all length and time scales. The relevant techniques include TEM,[10-16] synchrotron x-ray and neutron scattering, Raman and optical spectroscopy, [23, 24, 35] as well as scanning probe techniques such as atomic force microscopy, scanning tunneling microscopy, etc. [16]

The generic approach of reducing the size of active particles and shifting the burden of ion transport from through usually not-so-conductive electrode particles to through electrolyte media and across SEIs results in bigger influences of surface and interfaces on the battery performance, in intercalation and conversion electrodes alike. For example, small particle sizes and surface coating of $\mathrm{LiFePO}_{4}$ have enabled the adoption of the cathode compound for power battery applications. As the metastable solid solution phase in high electric fields is regarded 
mostly responsible for the fast ion transport, IOMs are particularly suited for elucidating such phenomena of the transient nature. [19**, 20, 22]

The complexity in conversion battery is rather different as it involves the diffusion of multiple ion species and the transformation of multiple lattice structures hence repeated creation, propagation, and annihilation of interfaces, which have been captured by several IOM studies. [11,36,37**] Figure 4 shows in situ TEM on Li ion transport and electrochemical reactions in $\mathrm{FeF}_{2}$. [13] In situ TEM is particularly powerful not only because of the high spatial resolution (down to sub-nm scale), but the analytical capabilities available within a single instrument, such as imaging, diffraction, spectroscopy and even holography, for multimodal measurements of the battery electrodes.[13,37] Combined IOMs uncover the surprisingly fast reaction rate within individual nanoparticles, in sharp contrast to the conventional belief of slow conversion reactions.[133] Results from in operando studies provide important insights in the design of new conversion electrodes of high power and energy density.[37] High vacuum requirement limits the convenient use of TEM as an IOM tool in battery research, solid state electrolytes (SSE) are often used in in situ TEM.

SSEs are considered a serious alternative to conventional liquid ones to address the safety and cycle life challenges, whereas they often suffer from low ionic conductivity, limiting the use of SSEs in power batteries.[7] Solid state batteries in planar deposited films are studied using SPM,[8] and micro-batteries with one dimension milled down to $<100 \mathrm{~nm}$ using focused ion beam for TEM studies. [155] Tracking ion transport and distribution within the electrolyte, electrode and at their interfaces reveals the importance of defects and interfaces on Li ion transport.[8,16] On the other hand, in situ neutron depth profiling (NDP) directly measures Li ion transport between two electrodes and across the electrolyte as the battery goes through 
charge/discharge cycles. $\left[23^{*}, 38,39\right]$ In situ NDP can also help identify electric leakage, soft shorting, and decomposition that can happen in SSEs in high power applications.[23]

\section{Safety and Long Life}

The safe use of RBs is the foremost requirement. However, the push to commercialize higher capacity batteries using more aggressive electrochemical reactions cause RBs operating ever closer to their stability limit, heightening the reliability and safety risks.[1,3,4,29] Fire and environmental hazard can occur in a catastrophic failure due to dendritic growth of active ions short-circuiting the opposite electrodes. On the other hand, long cycle and shelf life and high Coulombic efficiency (CE) directly impact the design capacity and cost requirements therefore their commercial viability. Those issues are all related to the undesirable transport and deposition of active ions. IOMs are particularly suited for critical studies of stray ions forming dendrites in safety issues, and the loss of active ions (e.g., lost wiring of electrode particles or trapped in parasitic reaction products) in capacity fading issues. By seeing ions, the onset of dendrite growth can be identified; by counting ions, new insights in the cycling CE can be obtained. The former is achievable by innovative imaging of ions using neutron,[40] x-ray and electronic and ion beam tomography,[41,42] MRI,[43] OM,[44,45], TEM,[46] etc., while the latter by neutron activation.[34]

Figure 5 shows recent studies by imaging Li distributions during the battery operation using MRI,[43] OM,[43] and neutron imaging (NI).[40] Ex situ 3D Li mapping using scanning neutron depth profiling (sNDP) is shown in Figure 5 (c). Although powerful, sNDP is limited by neutron flux and detection efficacy.[34*] As they all add valuable insights in the corresponding battery systems, limitations of individual technique vary from the lack of quantification and/or 
specification to the low spatial and/or time resolutions. There is a clear need for in operando, high resolution 3D imaging tools for visualizing the immobilization of active ions in undesirable processes.

NDP is highly specific and quantitative for Li detection and has reasonably good depth resolution for resolving electrode layers and interfaces. A unique use of current NDP capability is $1 \mathrm{D}$ depth profiling $\mathrm{Li}$ in electrodes to quantify three CEs other than the conventional roundtrip CE: for the ion/electron in single trips of charge and discharge, and the round trip of ions. They can be compared with the conventional high precision electric roundtrip CEs on charge/discharge cycling,[47] adding to our understanding of degradation processes. Together with comprehensive structural and compositional measurements, the complexity of battery system could be deconvoluted, and analytical models to interpret degradation be validated.[48]

\section{Alternative Systems and New Horizon}

To leap forward the RB technology, alternative approaches through the use of new materials, new reactions, and new platforms have been explored. One dominant force is the use of conversion reactions, in which anodic metal reacts with an unstable cathode, including metal fluoride, sulfur, oxygen, etc., and undergoes reversible atomic rearrangement on charge and discharge.[31*] Additional challenges arise from the complexity of conversion batteries, e.g., the mechanism and efficacy of catalytic oxygen reductions and oxygen evolution reactions in Li-O batteries.[49] New measurement techniques are needed to capture dramatic changes in both chemical compositions and morphology, as well as the transport of active ions. Another important new direction is hybridization of different energy storage systems. Examples include hybridizing intercalation and conversion, battery and capacitor, mass flow and intercalation, 
etc.[30] Multimodal IOMs, particularly with synergistic combination of techniques, are much needed to gain multitude information from the same system for correlation analysis. For example, researchers have used SPMs combined with confocal Raman spectroscopy,[35] and the transmission $\mathrm{x}$-ray microscopy combined with other x-ray scattering and spectroscopy methods,[22,50] to correlate morphology, structure and chemical states. Powerful commercial tools that combine light, electron and x-ray microscopy and measurements will change the course of materials research.[51] Such IOMs are critical for accelerating the development of RB technologies.

\section{Extreme Batteries}

Batteries for extreme condition applications usually play a very important role but often receive less attention. Those include specialized batteries for space, medicine, military, industry, infrastructure, and other applications. The requirements may vary drastically, from very stable and long life to high thermal tolerance to very light weight to radiation hardness etc. A space battery may require extreme longevity, but the degree of depth in charge/discharge can be very shallow. An implanted cardiovascular defibrillator battery needs to sustain harsh physiological environment and reliably deliver a precise electric pulse at exactly the right moment.[52] An ammunition detonation ignition battery is used once in its lifetime but needs to be stable for over 20 years, including storage and transport. IOMs for developing extreme batteries need to be compatible with extreme operational conditions, i.e., performing quantitative measurements on batteries undergoing relevant operations in special sample environments. Optical [35], neutron [53], magnetic [43], and other radiation-based techniques are particularly valuable for probing 
structures in electrodes behind thick walls, which are often needed to confine extreme physical conditions.

\section{Outlooks}

Rechargeable batteries are complex electrochemical systems, and operate often far from equilibrium and close to the instability limit. Many daunting challenges remain to be addressed to further advance the battery technology. Those include high energy/power density, high energy efficiency, long cycle life, safe to use, specialty batteries for extreme applications, etc. The rapid development of IOMs in recent years has greatly contributed to advancing battery sciences and engineering. As an example for the former, IOM studies have revealed phase transformation pathways in lithiating/delithiating $\mathrm{LiFePO}_{4}$; for the latter, General Electric Co has commercialized rechargeable sodium batteries with a cell design optimized using in operando synchrotron $x$-ray radiation. Many exciting new opportunities are on the horizon. (1) Advance IOMs with wider measurement ranges and higher detection resolutions, and on battery systems operating under realistic electrochemical and physical conditions. (2) Develop synergistic IOMs with multimodal measurement capabilities for simultaneously collecting multitude information from the same system for correlation analysis. (3) Develop higher temporal and spatial resolution IOMs especially those suited for 3D ion visualization and quantification, with advanced TEM and neutron techniques among the top of the list. Together they will play an essential role in developing next generation rechargeable batteries. 


\section{Acknowledgements}

HW acknowledges the financial support of NIST Award 70NANB12H238, and the use of the cold neutron facility at the NIST Center for Neutron Research. FW acknowledges the financial supports of the Laboratory Directed Research and Development (LDRD) program at Brookhaven National Laboratory, under Award No. 13-022, and U.S. Department of Energy (DOE) Office of Energy Efficiency and Renewable Energy under the Advanced Battery Materials Research (BMR) program, Contract No. DE-SC0012704. 
Figure 1

(a)
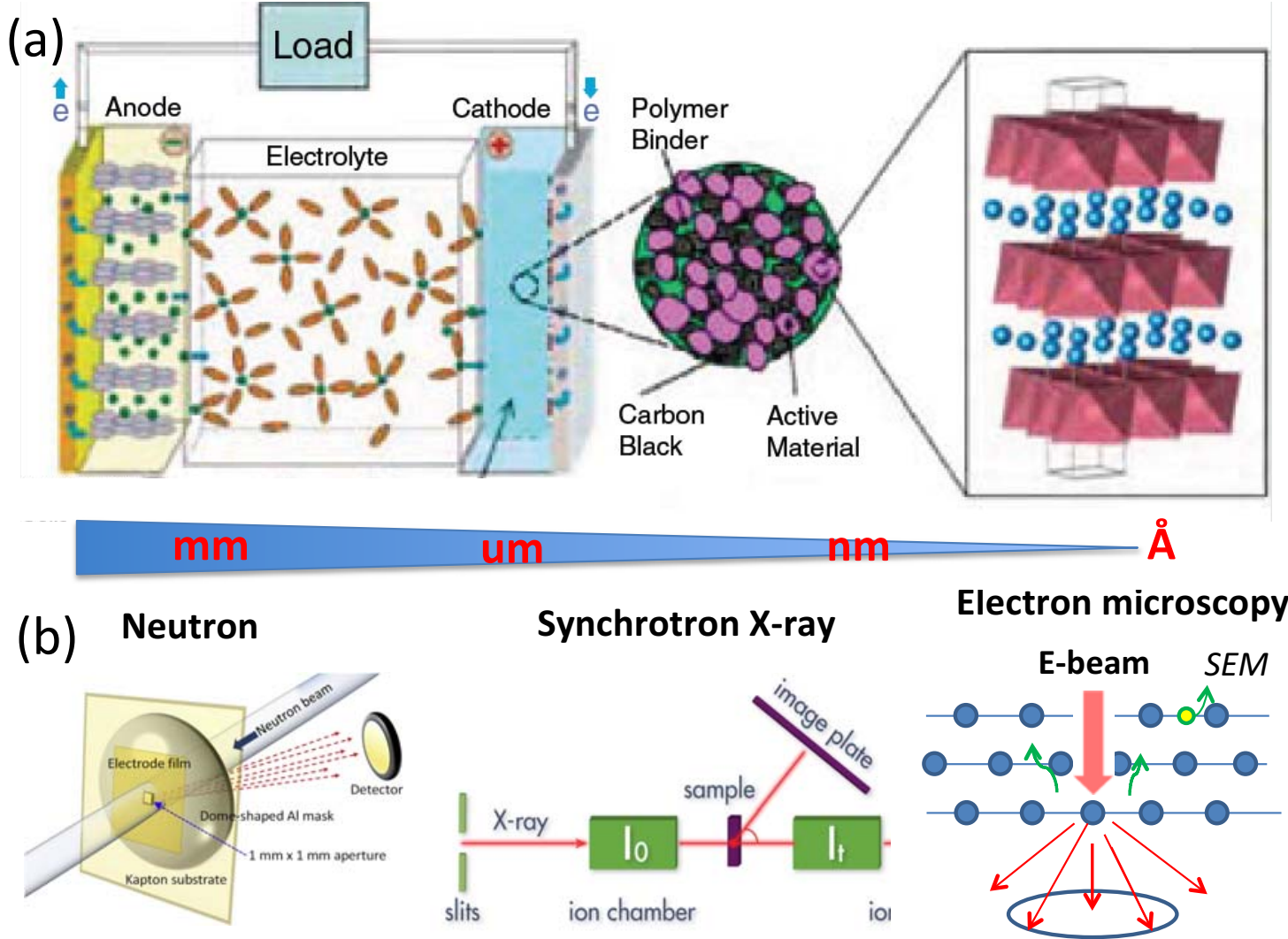

(S)TEM imaging, EELS

Figure 1: (a) Illustration of battery components at different scales. [4] From left to right, layered stacks of cathode and anode separated by electrolyte, with thickness of 10 s to $100 \mathrm{~s} \mu \mathrm{m}$; composite electrode with active material, carbon black, and polymer binder, with particle sizes of $10 \mathrm{~s} n$ to microns; lattice structures of active material with characteristic length of Angstroms.

(b) Corresponding in operando measurement techniques for probing structures at different length scales, as exemplified (from left to right) by neutron activation imaging, x-ray scattering, and election microscopy and spectroscopy. 


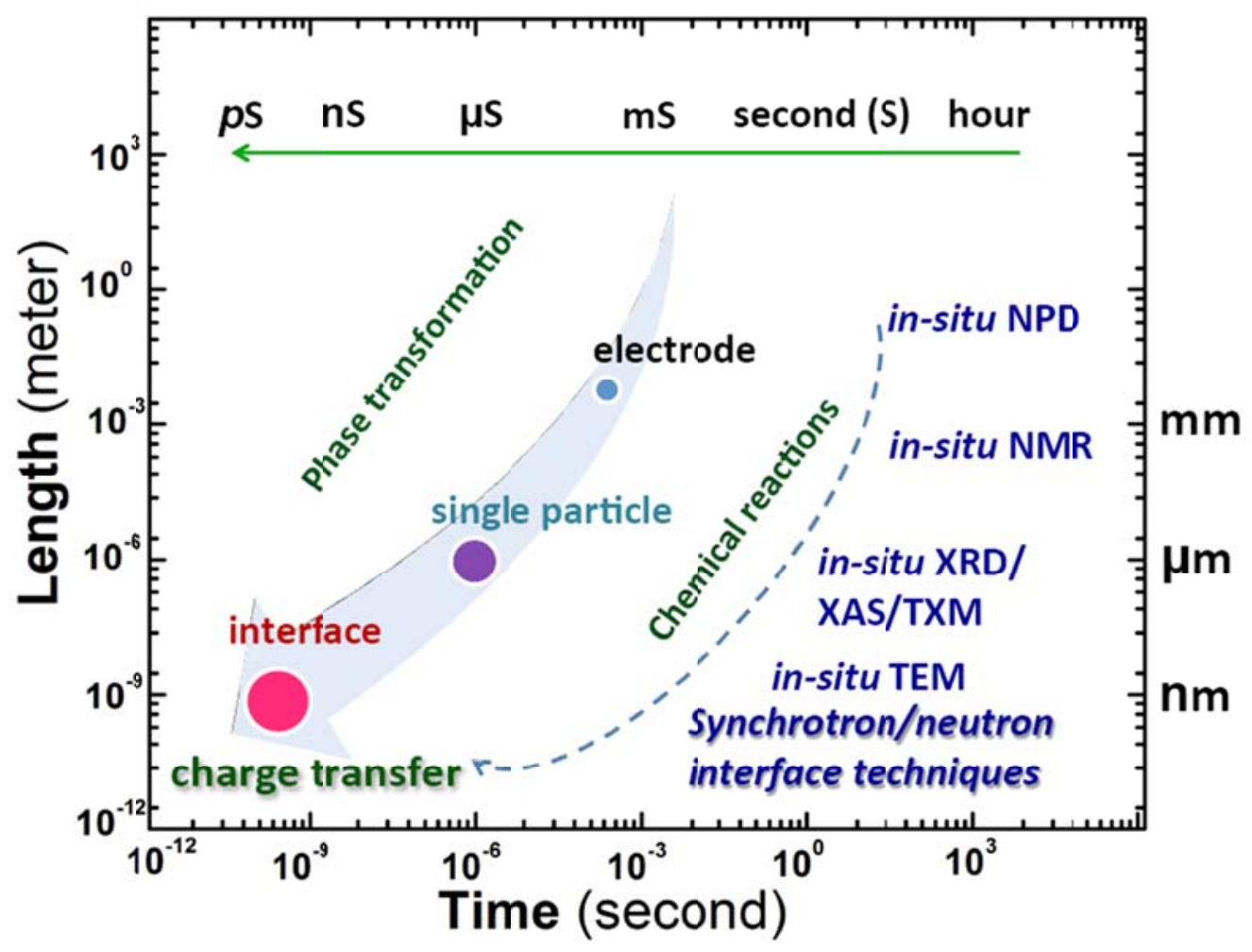

Figure. 2. Schematics of the time and length scales relevant to addressing RB issues. Proper in operando techniques are needed to match the time and length scales of the interest, covering the issues of the integrity and function of electrode layers, phase transformation and ion distributions in single or many particles, to the transport through and stability of interfaces and lattices.

Because of very different time and length scales in battery systems, complementary IOMs are used to gain a better understanding of the complexity. 
Figure. 3.

(a)

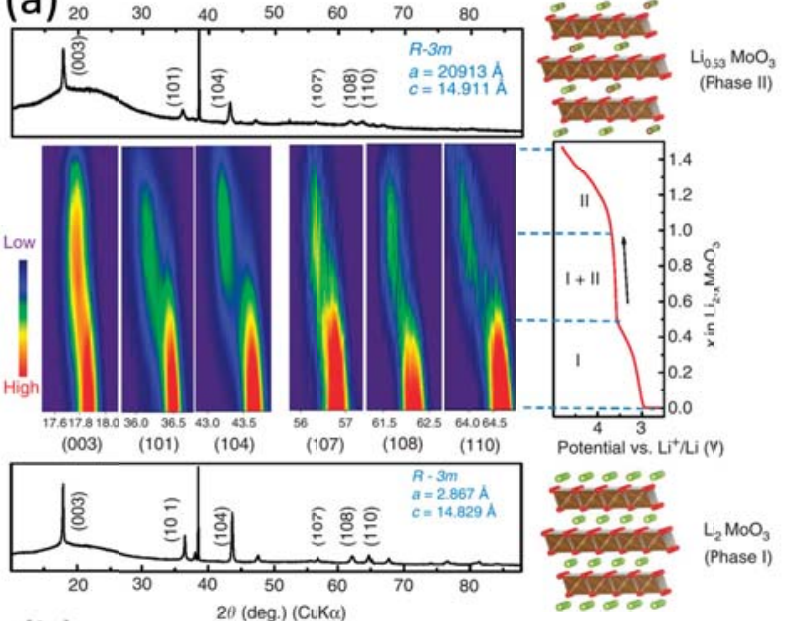

(b)

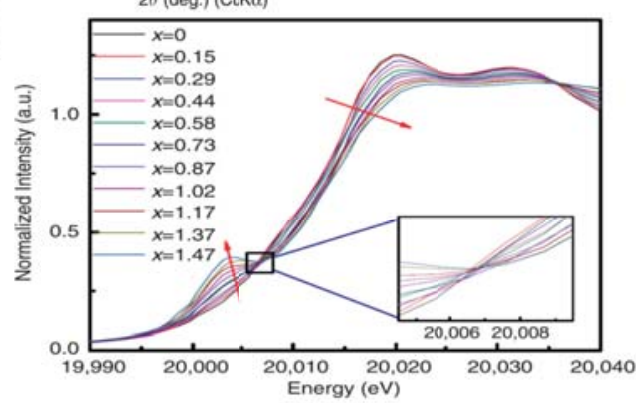

(c)

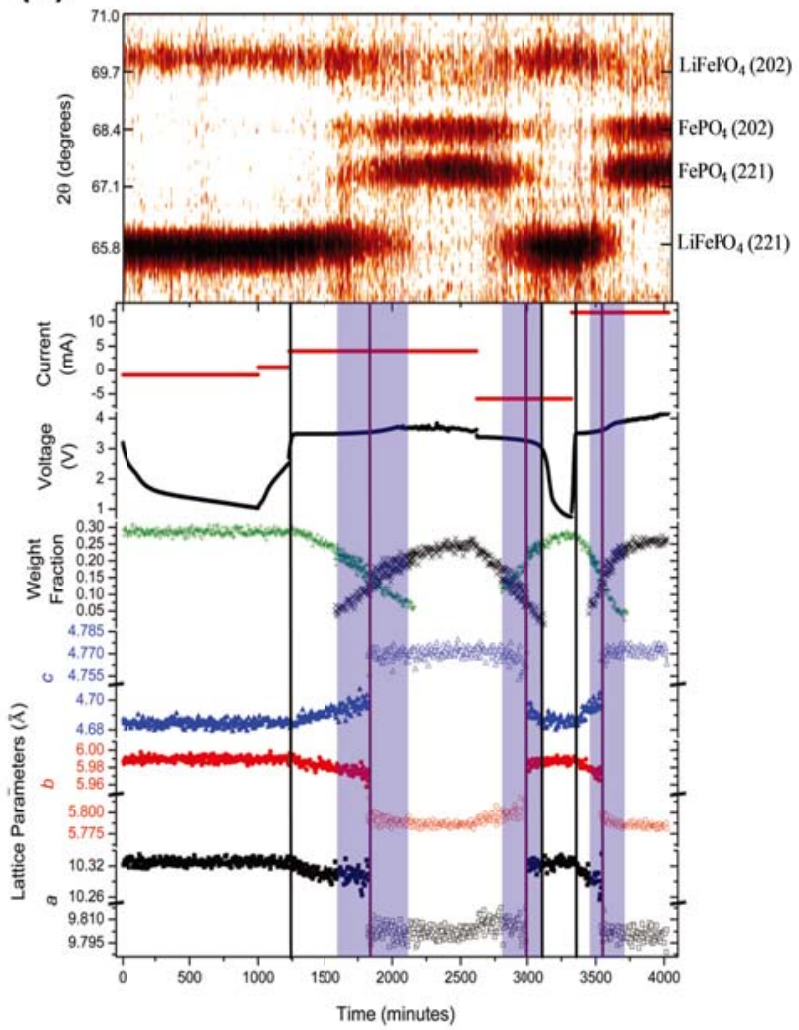

Figure 3. (a) In operando synchrotron x-ray diffraction (XRD) on $\mathrm{Li}_{2} \mathrm{MoO}_{3}$ cathode during first delithiation showing unusual lattice expansion with the movement and occupancy of ions. (b) In situ x-ray absorption near edge structure (XANES) spectra indicating the oxidation state of Mo ions. [11] (c) In situ neutron powder diffraction (NPD) on phase transformation in $\mathrm{LiFePO}_{4}$ cathodes. NPD data as a function of time as the battery goes through lithiation and delithiation cycles according to the applied current and corresponding voltage; data analysis and Rietveld refinements phase yield fractions of $\mathrm{LiFePO}_{4}$ (green crosses) and $\mathrm{FePO}_{4}$ (black crosses), as well as lattice parameters of $\mathrm{LiFePO}_{4}$ (solid symbols) and $\mathrm{FePO}_{4}$ (open symbols). [26 ] 
Figure 4
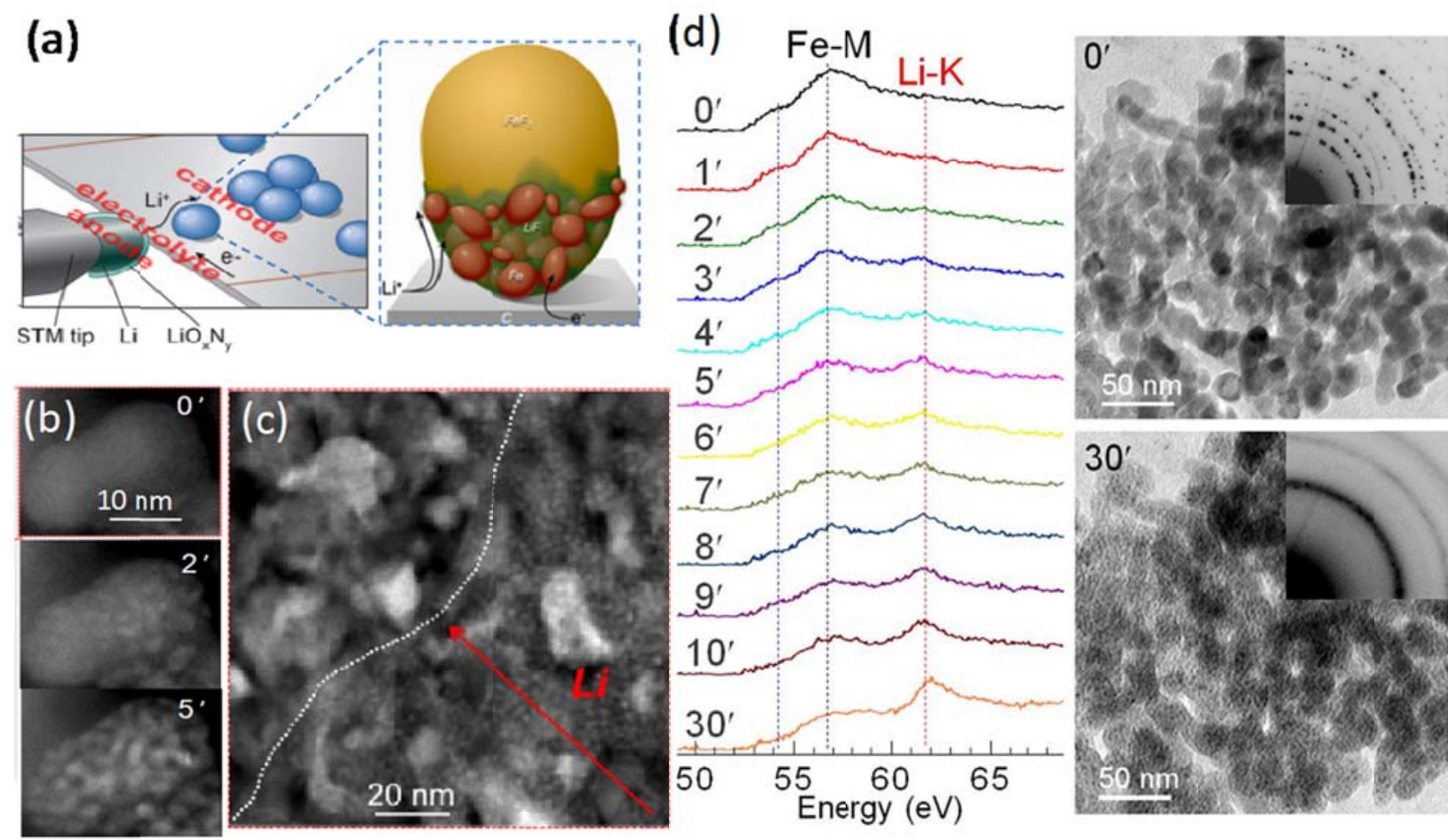

Figure 4. In situ tracking of electronic/ionic transport in $\mathrm{FeF}_{2}$ using the setup schematically shown in (a), within single particles and across thin films (b), and within agglomerates (c), using a combination of TEM imaging, diffraction, imaging, EELS within a same instrument. An illustration of the "layer-by-layer" conversion from $\mathrm{FeF}_{2}$ to $\mathrm{Fe}$ and $\mathrm{LiF}$ within a single particle (due to the limit of the electronic transport), was given in the inset of (a). [13] 
Figure 5

(a)
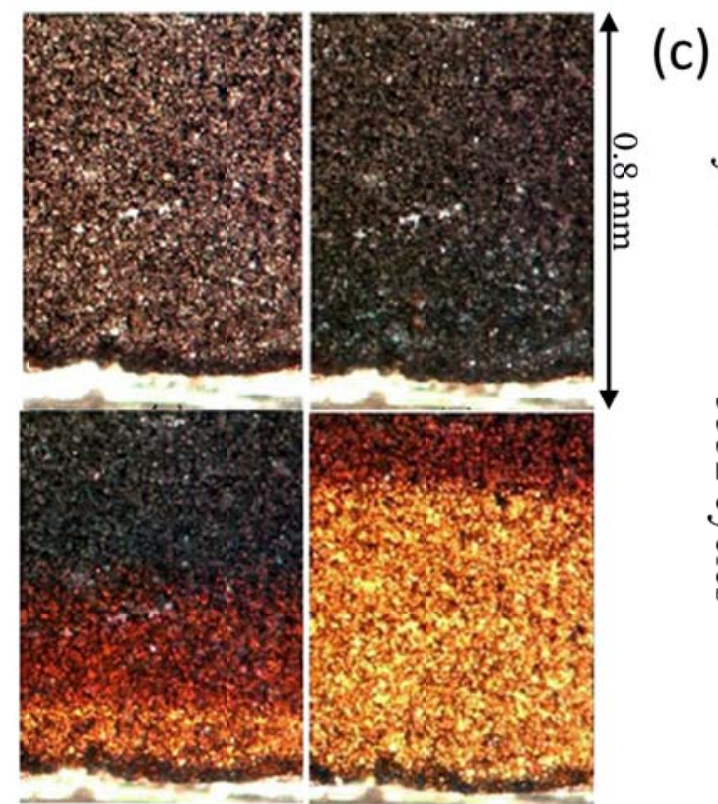
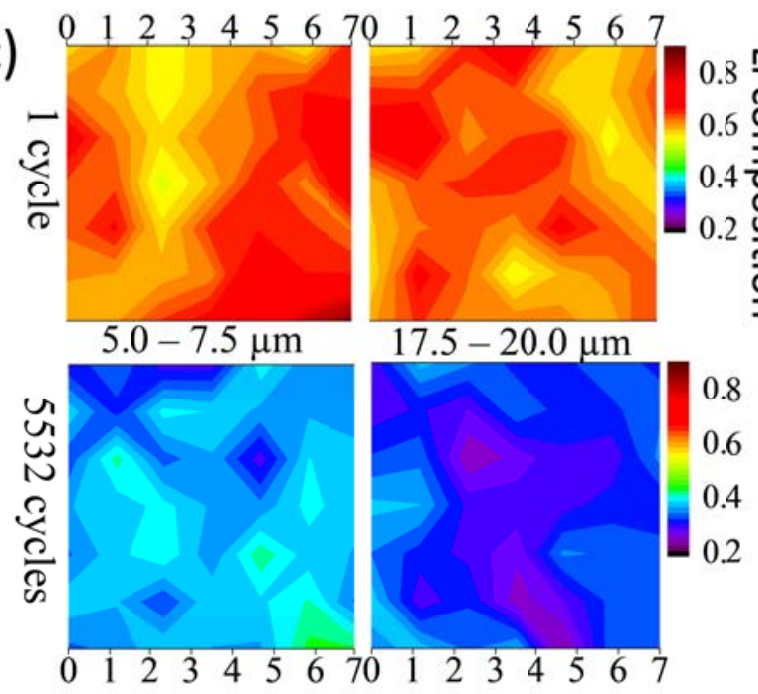

(d)

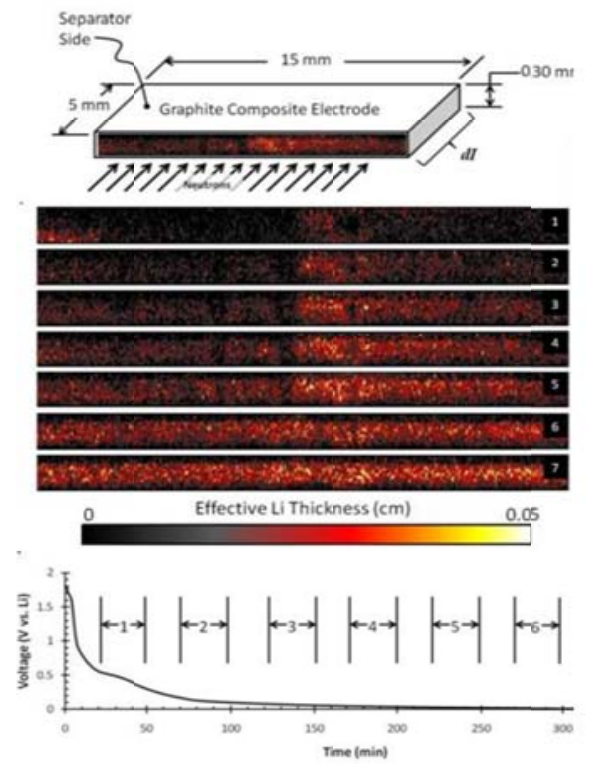

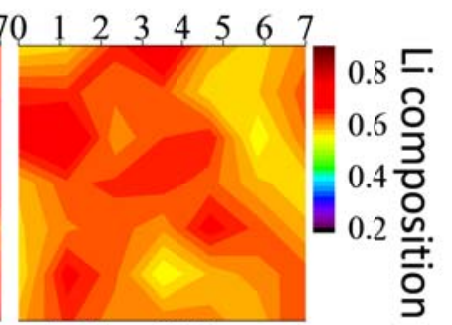

$17.5-20.0 \mu \mathrm{m}$

\section{8}

6 4 0.2 (b)
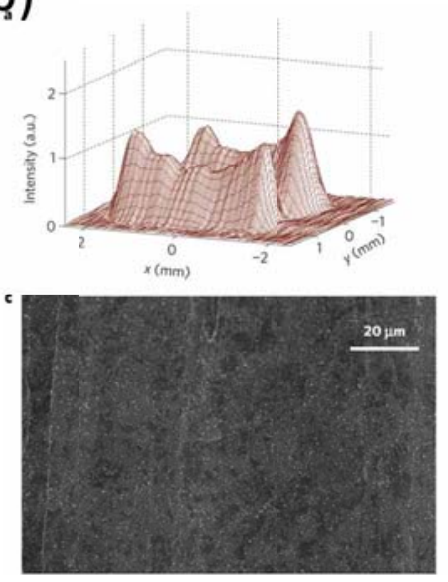
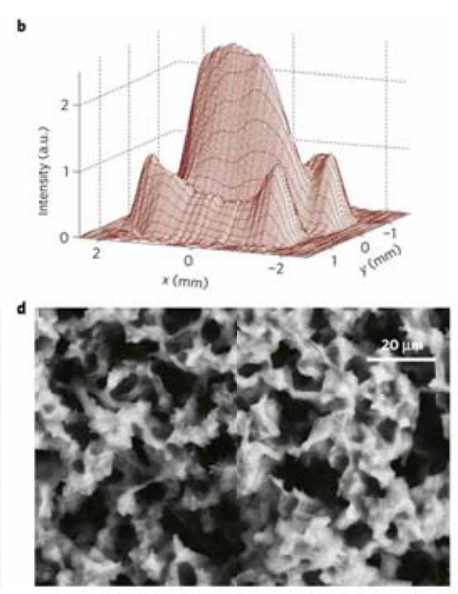
References:

[1] Whittingham MS: History, evolution, and future status of energy storage. Proceedings of the IEEE 2012, 100:1518-1534.

[2] Dunn B, Kamath H, Tarascon JM: Electrical energy storage for the grid: a battery of choice. Science 2011, 334:928-935.

[3] Goodenough J, Abruna HD, Buchanan MV: Basic Research Needs for Electrical Energy

Storage: Report of the Basic Energy Science Work- shop on Electrical Energy Storage; U.S.

Department of Energy: Washington, DC, 2007.

[4] Whittingham MS: Materials challenges facing electrical energy storage. MRS Bulletin 2008, 33:411-419.

**[5] Dudney NJ, Li J: Using all energy in a battery. Science 2015, 347:131-132. Elucidating the importance of electric and ionic wiring design for achieving the theoretical capacity of batteries.

*[6] Kirshenbaum K, Bock DC, Lee CY, Zhong Z, Takeuchi KJ, Marschilok AC, Takeuchi ES: In situ visualization of $\mathrm{Li} / \mathrm{Ag}_{2} \mathrm{VP}_{2} \mathrm{O}_{8}$ batteries revealing rate-dependent discharge mechanism. Science 2015, 347:149-154. Using in situ and ex situ techniques to help design electrodes with the lower polarization and the better rate performance.

[7] Dudney NJ, Nanda J, West WC: Handbook of Solid State Batteries. World Scientific; 2015. *[8] Harks PPRML, Mulder FM, Notten PHL: In situ methods for Li-ion battery research: A review of recent developments. Journal of Power Sources 2015, 288:92-105. Providing a comprehensive summary of in situ measurement tools for Li-ion battery research.

[9] Nelson Weker J, Toney MF: Emerging In Situ and Operando Nanoscale X-Ray Imaging Techniques for Energy Storage Materials. Advanced Functional Materials 2015, 25:16221637.

*[10] Wang CM: In situ transmission electron microscopy and spectroscopy studies of rechargeable batteries under dynamic operating conditions: A retrospective and perspective view, J. Mater. Res., 2015, 30:326-339. Providing an overall and in-depth review of the in situ TEM techniques for battery research.

[11] He K, Zhang S, Li J, Yu XQ, Meng QP, Zhu YZ, Hu EY, Sun K, Yun H, Yang XQ, et al:

Visualizing non-equilibrium lithiation of spinel oxide via in situ transmission electron microscopy. Nature communications 2016, 7: No. 11441.

[12] Wang CM, Li XL; Wang ZG; Xu W; Liu J; Gao F; Kovarik L; Zhang JG; Howe J; Burton DJet al. In situ TEM investigation of congruent phase transition and structural evolution of nanostructured silicon/carbon anode for lithium ion batteries. Nano letters 2012, 12: 16241632.

[13] Wang F, Yu HC; Chen MH; Wu LJ; Pereira N; Thornton K; Van der Ven A; Zhu YM; Amatucci GG; Graetz J: Tracking lithium transport and electrochemical reactions in nanoparticles. Nature communications 2012, 3:1201.

[14] Wen Y, He K, Zhu Y, Han F, Xu Y, Matsuda I, Ishii Y, Cumings J, Wang CS: Expanded

graphite as superior anode for sodium-ion batteries. Nature communications 2014, 5:4033.

[15] Wang Z, Santhanagopalan D, Zhang W, Wang F, Xin HL, He K, Li J, Dudney N, Meng YS: In situ STEM/EELS Observation of Nanoscale Interfacial Phenomena in All-Solid-State Batteries. Nano Letters 2016, 16:6b01119. 
[16] Sacci, RL, Black JM; Balke N; Dudney NJ; More KL; Unocic RR: Nanoscale Imaging of Fundamental Li Battery Chemistry: Solid-Electrolyte Interphase Formation and Preferential Growth of Lithium Metal Nanoclusters. Nano Letts 2015, 15:2011-2018. [17] v. Cresce A, Russell SM, Baker DR, Gaskell KJ, Xu K: In Situ and Quantitative Characterization of Solid Electrolyte Interphases. Nano Letts. 2014, 14:1405-1412. [18] Blanc F, Leskes M, Grey CP: In situ solid-state NMR spectroscopy of electrochemical cells: batteries, supercapacitors, and fuel cells. Accounts of chemical research 2013, 46:19521963.

**[19] Liu H, Strobridge FC, Borkiewicz OJ, Wiaderek KM, Chapman KW, Chupas PJ, Grey CP: Capturing metastable structures during high-rate cycling of LiFePO4 nanoparticle electrodes. Science 2014, 344:1252817. In situ x-ray diffraction with detailed data analysis to reveal the nonequilibrium metastable solid solution phase during high rate cycling. [20] Chapman KW: Emerging operando and $x$-ray pair distribution function methods for energy materials development. MRS Bulletin 2016, 41:231-240.

[21] Ulvestad A, Singer A, Clark JN, Cho HM, Kim JW, Harder R, Maser J, Meng YS, Shpyrko OG: Topological defect dynamics in operando battery nanoparticles. Science 2015, 348:1344-1347.

[22] Wang J, Chen-Wiegart YCK, Wang J: In operando tracking phase transformation evolution of lithium iron phosphate with hard X-ray microscopy. Nature communications 2014, 5:4570

*[23] He Y, Wang H: In situ neutron techniques for lithium ion and solid-state rechargeable batteries. in Handbook of Solid State Batteries World Scientific 2015, 51-77. Providing an overview of in situ neutron techniques emphasizing both the experimental realization and applicable battery issues.

[24] Sharma N, Wagemaker M: Lithium-ion batteries. In Neutron Applications in Materials for Energy, Springer International Publishing, 2015, 139-203.

[25] DeCaluwe SC, Dhar BM, Huang L, He Y, Yang K, Yang B, Owejan J, Zhao Y, Talin AA, Dura JA, Wang H., Pore collapse and regrowth in silicon electrodes for rechargeable batteries, Phys Chem Chem Phys, 2015, 17:11301-11312.

[26] Sharma N, Guo X, Du G, Guo Z, Wang J, Wang, Peterson VK: Direct evidence of concurrent solid-solution and two-phase reactions and the nonequilibrium structural evolution of LiFePO 4 . J. Am. Chem. Soc. 2012, 134:7867-7873.

[27] Xu K: Electrolytes and interphases in Li-ion batteries and beyond. Chemical reviews 2014, 114:11503-11618.

[28] Zhou YN, Ma J, Hu E, Yu X, Gu L, Nam KW, Chen L, Wang Z, Yang XQ: Tuning charge-discharge induced unit cell breathing in layer-structured cathode materials for lithium-ion batteries. Nature communications 2014, 5:5381.

[29] Whittingham MS: Ultimate limits to intercalation reactions for lithium batteries. Chemical Reviews, 2014, 114:11414-11443.

*[30] Choi JW, Aurbach D: Promise and reality of post-lithium-ion batteries with high energy densities. Nature Reviews Materials, 2016, 1:16013. Offering an insightful overview of the future rechargeable battery technologies.

*[31] Andre D, Kim SJ, Lamp P, Lux SF, Maglia F, Paschos O, Stiaszny B: Future generations of cathode materials: an automotive industry perspective. Journal of Materials Chemistry A 
2015, 3:6709-6732. Providing the issues and goals of rechargeable battery technology from industrial perspectives, which would offer a strong guidance to battery research.

[32] Sougrati MT, Darwiche A, Liu X, Mahmoud A, Hermann RP, Jouen S, Monconduit L, Dronskowski R, Stievano L, Transition-Metal Carbodiimides as Molecular Negative Electrode Materials for Lithium- and Sodium-Ion Batteries with Excellent Cycling Properties, Angew, Chem, Int. Ed, 2016, 55:5090-5095.

[33] Li Y, Meyer S, Lim J, Lee SC, Gent WE, Marchesini S, Krishnan H, Tyliszczak T, Shapiro D, Kilcoyne AL, Chueh WC, Effects of particle size, electronic connectivity, and incoherent nanoscale domains on the sequence of lithiation in $\mathrm{LiFePO}_{4}$ porous electrodes. Advanced Materials 2015, 27:6591-6597.

*[34] He Y, Downing RG, Wang H: 3D mapping of lithium in battery electrodes using neutron activation. Journal of Power Sources 2015, 287:226-230. Illustrating the use of a unique technique, the scanning neutron depth profiling, to reveal heterogeneous Li distribution in battery electrodes.

[35] Stancovski V, Badilescu S. In situ Raman spectroscopic-electrochemical studies of lithium-ion battery materials: a historical overview. Journal of Applied Electrochemistry 2014, 44:23-43.

[36] Hu YY, Liu Z, Nam KW, Borkiewicz OJ, Cheng J, Hua X, Dunstan MT, Yu X, Wiaderek $\mathrm{KM}$, Du LS, et al. Origin of additional capacities in metal oxide lithium-ion battery electrodes. Nature Materials 2013, 12:1130-1136.

**[37] Wang F, Kim SW, Seo DH, Kang K, Wang L, Su D, Vajo JJ, Wang J, Graetz J: Ternary metal fluorides as high-energy cathodes with low cycling hysteresis. Nature Communications 2015. 6:6668. Combining in situ and ex situ synchrotron XRD, XAS, STEM and EELS with electrochemical measurements to reveal a reversible conversion redox reaction.

[38] Liu DX, Wang J, Pan K, Qiu J, Canova M, Cao LR, Co AC: In situ quantification and visualization of lithium transport with neutrons. Angewandte Chemie International Edition 2014, 53:9498-9502.

[39] Liu DX, Co AC, Revealing chemical processes involved in electrochemical (de)lithiation of Al with in situ neutron depth profiling and x-ray diffraction, J. Am. Chem. Soc., 2016, 138:231-238.

[40] Owejan JP, Gagliardo JJ, Harris SJ, Wang H, Hussey DS, Jacobson DL: Direct measurement of lithium transport in graphite electrodes using neutrons. Electrochimica Acta 2012, 66:94-99.

[41] Ebner M, Marone F, Stampanoni M, Wood V: Visualization and quantification of electrochemical and mechanical degradation in Li ion batteries. Science 2013, 342: 716-720. [42] Shearing PR, Howard LE, Jørgensen PS, Brandon NP, Harris SJ: Characterization of the 3-dimensional microstructure of a graphite negative electrode from a $\mathrm{Li}$-ion battery. Electrochemistry communications 2010, 12:374-377.

[43] Chandrashekar S, Trease NM, Chang HJ, Du LS, Grey CP, Jerschow A: 7Li MRI of Li batteries reveals location of microstructural lithium. Nature Materials 2012, 11:311-315. [44] Harris SJ, Timmons A, Baker DR, Monroe C: Direct in situ measurements of Li transport in Li-ion battery negative electrodes. Chemical Physics Letters 2010, 485:265-274. [45] Love CT, Baturina OA, Swider-Lyons KE: Observation of Lithium Dendrites at Ambient Temperature and Below. ECS Electrochem. Lett, 2015, 4:A24-A27. 
[46] Sacci RL. Dudney NJ, More KL, Parent LR, Arslan I, Browning ND, Unocic RR: Direct visualization of initial SEI morphology and growth kinetics during lithium deposition by in situ electrochemical transmission electron microscopy. Chem. Comm, 2014, 50:2104-2107. [47] Smith AJ, Burns JC, Trussler S, Dahn JR: Precision measurements of the coulombic efficiency of lithium-ion batteries and of electrode materials for lithium-ion batteries. $J$. Electrochem. Soc. 2010, 157:A196-A202.

[48] Huggins RA: Advanced Batteries: Material Science Aspects, Springer, New York, 2009, p.419

[49] Lu YC, Betar MG, Kwabi DG, Harding JR, Mitchell RR, Whittingham MS, Yang SH:

Lithium-oxygen batteries: bridging mechanistic understanding and battery performance. Energy \& Environmental Science 2013, 6:750-768.

[50] Bauer S, de Biasi L, Glatthaar S, Toukam L, Gesswein H, Baumbach T: In operando study of the high voltage spinel cathode material $\mathrm{LiNi}_{0.5} \mathrm{Mn}_{1.5} \mathrm{O}_{4}$ using two dimensional full-field spectroscopic imaging of Ni and Mn. Phys Chem Chem Phys, 2015, 17:16388-16397.

[51] http://www.zeiss.com/microscopy/en_de/solutions/materials-science/battery-research.html [52] Bock DC, Marschilok AC, Takeuchi KJ, Takeuchi ES: Batteries used to power implantable biomedical devices. Electrochimica acta 2012, 84:155-164.

[53]Taminato S, Yonemura M, Shiotani S, Kamiyama T, Torii S, Nagao M, Ishikawa Y, Mori K, Fukunaga T, et al.: Real-time observations of lithium battery reactions-operando neutron diffraction analysis during practical operation, Nature Scientific Reports, 2016, 6, 28843. 